\title{
Cannabinoids reduce ErbB2-driven breast cancer progression through Akt inhibition
}

María M Caffarel 1,6, Clara Andradas', Emilia Mira², Eduardo Pérez-Gómez , Camilla Cerutti ${ }^{1,6}$, Gema Moreno-Bueno $^{3}$, Juana M Flores ${ }^{4}$, Isabel García-Real ${ }^{4}$, José Palacios ${ }^{5}$, Santos Mañes ${ }^{2}$, Manuel Guzmán ${ }^{1}$, Cristina Sánchez ${ }^{1 *}$

\begin{abstract}
Background: ErbB2-positive breast cancer is characterized by highly aggressive phenotypes and reduced responsiveness to standard therapies. Although specific ErbB2-targeted therapies have been designed, only a small percentage of patients respond to these treatments and most of them eventually relapse. The existence of this population of particularly aggressive and non-responding or relapsing patients urges the search for novel therapies. The purpose of this study was to determine whether cannabinoids might constitute a new therapeutic tool for the treatment of ErbB2-positive breast tumors. We analyzed their antitumor potential in a well established and clinically relevant model of ErbB2-driven metastatic breast cancer: the MMTV-neu mouse. We also analyzed the expression of cannabinoid targets in a series of 87 human breast tumors.
\end{abstract}

Results: Our results show that both $\Delta^{9}$-tetrahydrocannabinol, the most abundant and potent cannabinoid in marijuana, and JWH-133, a non-psychotropic $\mathrm{CB}_{2}$ receptor-selective agonist, reduce tumor growth, tumor number, and the amount/severity of lung metastases in MMTV-neu mice. Histological analyses of the tumors revealed that cannabinoids inhibit cancer cell proliferation, induce cancer cell apoptosis, and impair tumor angiogenesis.

Cannabinoid antitumoral action relies, at least partially, on the inhibition of the pro-tumorigenic Akt pathway. We also found that $91 \%$ of ErbB2-positive tumors express the non-psychotropic cannabinoid receptor $\mathrm{CB}_{2}$.

Conclusions: Taken together, these results provide a strong preclinical evidence for the use of cannabinoid-based therapies for the management of ErbB2-positive breast cancer.

\section{Background}

Breast cancer represents approximately $30 \%$ of newly diagnosed cancers each year. Almost one third of them overexpresses the ErbB2 tyrosine kinase receptor (Her2 in humans, Neu in rats), a member of the EGF receptor family [1]. Phosphorylation of their intracellular domains upon engagement by their ligands induce receptor homo- or heterodimerization, leading to the activation of key signaling pathways that promote cell proliferation and survival, including the phosphatidylinositol 3-kinase (PI3K)/Akt pathway and the ERK/MAPK cascade. Although no specific ligand for ErbB2 has been identified yet, this receptor is the preferred heterodimerization partner of the family [2]. ErbB2-overexpressing breast tumors are characterized by very aggressive clinical courses and decreased survival rates, mostly due to the

\footnotetext{
* Correspondence: cristina.sanchez@quim.ucm.es
'Dept. Biochemistry and Molecular Biology I, School of Biology, Complutense

* Correspondence: cristina.sanchez@quim.ucm.es
'Dept. Biochemistry and Molecular Biology I, School of Biology, Complutense University, Madrid, Spain
}

(c) 2010 Caffarel et al; licensee BioMed Central Ltd. This is an Open Access article distributed under the terms of the Creative Commons Attribution License (http://creativecommons.org/licenses/by/2.0), which permits unrestricted use, distribution, and reproduction in any medium, provided the original work is properly cited. characteristics make ErbB2-overpressing tumors less responsive to conventional therapies. One of the most recent advances in the treatment of these tumors is the use of a humanized neutralizing monoclonal antibody against ErbB2 (Trastuzumab) [3]. Although this strategy has been very successful, around $75 \%$ of patients with ErbB2-overexpressing tumors do not respond to Trastuzumab, and nearly $15 \%$ of the responders eventually develop metastases [4]. The existence of this considerable population of non-responding and relapsing patients urges the search for novel treatments.

The therapeutic potential of cannabinoids, the active compounds of marijuana and their derivatives, has been known for centuries. There is increasing evidence supporting that they might be beneficial in various pathological contexts such as pain, inflammation, eating disorders, and brain damage, amongst others $[5,6]$. 
Cannabinoids exert most of their actions by binding to and activating specific $G$ protein-coupled receptors. To date, two cannabinoid receptors, namely $\mathrm{CB}_{1}$ and $\mathrm{CB}_{2}$, have been cloned and characterized from mammalian tissues, the main difference between them being their tissue expression pattern. Thus, while $\mathrm{CB}_{1}$ receptors are ubiquitously located, with their highest presence found in the central nervous system, $\mathrm{CB}_{2}$ receptor expression is mostly restricted to particular elements of the immune system $[5,6]$. During the last decade, evidence has accumulated suggesting that cannabinoids might be useful for the treatment of cancer. These compounds exert anti-proliferative, pro-apoptotic, anti-angiogenic, and anti-invasive effects in different cell-culture and animal models of cancer $[7,8]$. Here, we used a genetically engineered animal model of ErbB2-driven metastatic breast cancer (the MMTV-neu mouse) to analyze the antitumoral potential of cannabinoids in this particularly aggressive pathology. These animals express the rat ErbB2 oncogene (neu) under the control of the hormone-sensitive mouse mammary tumor virus-long terminal repeat (MMTV-LTR) promoter [9]. Selective overexpression of neu in the mammary epithelium results in the spontaneous development of focal mammary tumors after a long latency (5-12 months) [9]. Results presented herein (i) show that ErbB2-positive invasive human breast tumors express $\mathrm{CB}_{2}$ receptors, (ii) demonstrate that $\Delta^{9}$-tetrahydrocannabinol (THC) and the non-psychotropic $\mathrm{CB}_{2}$ cannabinoid receptor agonist JWH-133 significantly reduce tumor progression in a clinically relevant model of ErbB2-positive metastatic breast cancer, and (iii) shed light on the mechanism of cannabinoid antitumoral action in vivo.

\section{Results}

\section{Human ErbB2-positive breast tumors express $\mathrm{CB}_{2}$} cannabinoid receptors

We first analyzed whether ErbB2-positive human breast tumors express cannabinoid targets (i.e. cannabinoid receptors). We performed an immunohistochemical analysis of $C_{1}$ and $C_{2}$ receptors in 87 grade 3 invasive breast ductal carcinomas and 6 non-tumoral mammary samples by tissue microarrays. $\mathrm{CB}_{1}$ immunoreactivity was detected in only $14 \%$ of the tumors $(12 / 87)$, and no correlation was found between this receptor expression and ErbB2 expression ( $\mathrm{p}=0.198$, Fig. 1). Conversely, $\mathrm{CB}_{2}$ receptor staining was evident in $72 \%$ of the carcinomas (63/87) and it was significantly associated with ErbB2 expression, since it was observed in $91 \%$ of the ErbB2-positive tumors (21/23, $\mathrm{p}=0.018$, Fig. 1). Moreover, we detected no significant $\mathrm{CB}_{1}$ or $\mathrm{CB}_{2}$ receptor immunoreactivity in non-transformed mammary tissue (data not shown).

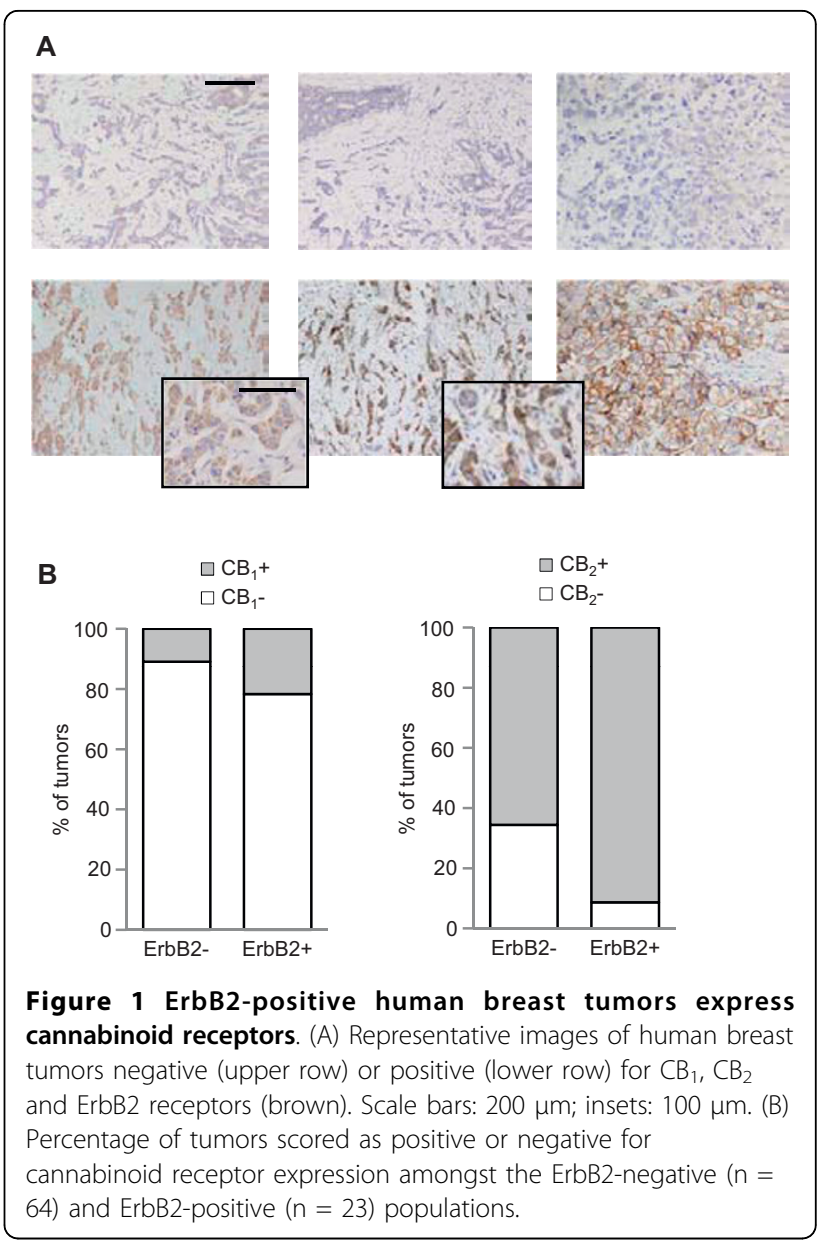

\section{Cannabinoids exert an antitumoral effect in the MMTV-} neu model of breast cancer

We then analyzed the effect of cannabinoids on tumor progression in a well established and clinically relevant animal model of ErbB2-driven metastatic breast cancer, the MMTV-neu mouse. We first observed that our MMTV-neu colony develops breast tumors after a long latency period similar to that previously reported [9]. In particular, $50 \%$ of the females had tumors by week 36 (Additional file 1: Fig. S1A). Overexpression of the rat ErbB2 transgene (neu) in the tumors was verified by real-time quantitative PCR (Additional file 1: Fig. S1B). Treatment with cannabinoids, either THC, the main marijuana-derived cannabinoid in terms of abundance and potency, or JWH-133, a synthetic $\mathrm{CB}_{2}$ receptorselective agonist, strongly slowed down tumor growth (Fig. 2A), leading to smaller lesions at the end of the treatment (Additional file 1: Fig. S2). These compounds, however, did not change the histomorphologic features of the tumors. Thus, the three different experimental groups generated focal, ductal, solid, well vascularized mammary tumors surrounded by a non-invasive 

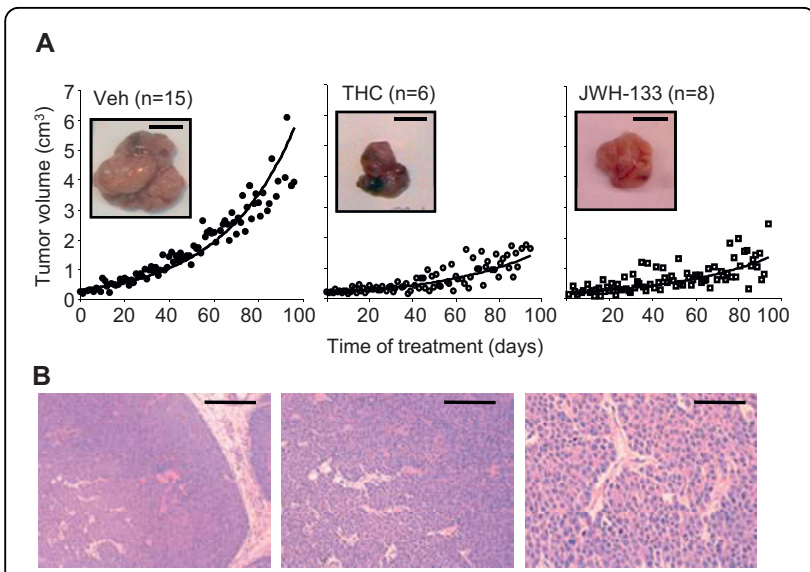

C

\begin{tabular}{ccccc}
$\begin{array}{c}\text { Tumors/ } \\
\text { animal }\end{array}$ & 1 & 2 & 3 & 4 or more \\
\hline $\begin{array}{c}\text { Control } \\
(\mathrm{n}=12)\end{array}$ & $25 \%$ & $8 \%$ & $25 \%$ & $41 \%$ \\
$\begin{array}{c}\mathrm{THC} \\
(\mathrm{n}=6)\end{array}$ & $50 \%$ & $17 \%$ & $33 \%$ & $0 \%$ \\
$\begin{array}{c}\mathrm{JWH}-133 \\
(\mathrm{n}=6)\end{array}$ & $33 \%$ & $50 \%$ & $17 \%$ & $0 \%$ \\
\hline
\end{tabular}

D
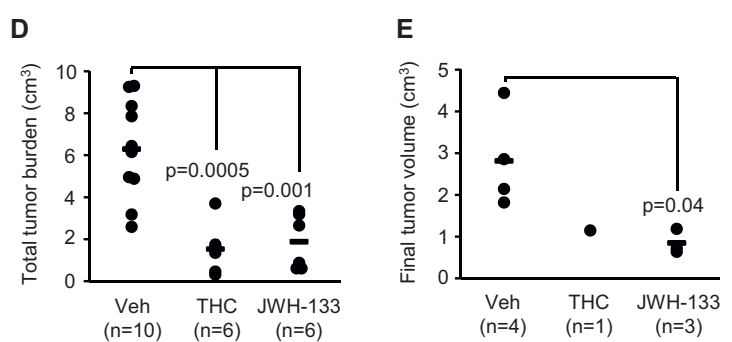

Figure 2 Cannabinoids inhibit breast tumor growth in vivo and the number of tumors generated per animal. (A) Volume timecourse (scale bar: $1 \mathrm{~cm}$ ) of the first tumor appeared in each animal. (B) Representative images (H\&E staining) of the histopathology of the MMTV-neu-derived mammary tumors. Scale bars (from left to right): $200 \mu \mathrm{m}, 100 \mu \mathrm{m}$ and $50 \mu \mathrm{m}$. (C) Percentage of animals with $1,2,3,4$ or more tumors at the end of the treatment (90 days) in each experimental group. (D) Total tumor burden (total tumor volume per animal) determined 90 days after cannabinoid or vehicle treatment. (E) Volume of the tumors appeared in second, third or subsequent positions, 40 days after their appearance. The small size of the cannabinoid-treated groups is due to the very few second or third tumors appeared early enough to last 40 days in the animals before the end of the treatment ( 90 days after the appearance of the first tumor).

hyperplasic mammary epithelium (Fig. 2B). We also observed that MMTV-neu-derived tumors express $\mathrm{CB}_{1}$ and $\mathrm{CB}_{2}$ cannabinoid receptor mRNA (determined by real-time quantitative $\mathrm{PCR}$, data not shown) and protein (Additional file 1: Fig. S3).

Of interest, cannabinoids not only impaired tumor growth, but also blocked tumor generation per se. Thus, while $41 \%$ of vehicle-treated animals developed 4 or more tumors (up to 6), cannabinoid-treated animals never developed more than 3 tumors (Fig. $2 \mathrm{C}, \mathrm{p}<0.05$ ). Consequently, total tumor burden was strikingly decreased by cannabinoids (Fig. 2D). There was also a delay in the appearance of the subsequent tumors in these animals. Thus, the average latency for the generation of a second tumor in vehicle-treated, THC-treated and JWH-133-treated animals was 33, 46 and 54 days, respectively. As mentioned in the Methods section, only the first tumor in each animal was treated peritumorally with cannabinoids. However, we detected a remarkable growth-inhibitory effect of cannabinoids in those tumors appeared in second place (Fig. 2E).

\section{Cannabinoids impact tumor cell proliferation, tumor cell survival and tumor angiogenesis}

We next analyzed the proliferative potential of cancer cells and found that it was reduced by both THC and JWH-133, as indicated by a decreased number of Ki67positive cells in cannabinoid-treated tumors (Fig. 3A). Cannabinoid administration also increased the number of cleaved (active) caspase 3-positive cells within the tumors, indicating that these compounds induce cancer cell death by apoptosis (Fig. 3B). Tumor vascularization was also impaired by cannabinoids, as both THC and JWH-133 decreased the number of blood vessels in the tumors, as determined by CD31 staining (Fig. 3C). To evaluate the possible contribution of the immune response to cannabinoid antitumoral action, we analyzed by immunofluorescence the degree of immune infiltration in the tumors. The percentage of CD45-positive cells (differentiated hematopoietic cells except erythrocytes and platelets) within the tumors was very low in all the samples tested and no significant differences between experimental groups were detected (Fig. 3D). These data suggest that cannabinoid treatment does not affect the infiltration of immune cells into the tumor parenchyma.

\section{Cannabinoids decrease breast cancer metastases in the lungs}

It has been previously reported that a high percentage of tumor-bearing MMTV-neu animals develop metastases in the lungs [9]. Specifically, we detected lung metastases (Fig. 4A) in 67\% of our vehicle-treated MMTVneu animals (Fig. 4B). The cell morphology, tumor architecture, and overexpression of the neu transgene mRNA in these lung structures confirmed the metastatic nature of the lesions (Figs. S1C and D). THC reduced the percentage of animals with lung metastases (Fig. 4B). Although JWH-133 did not decrease this proportion, it significantly reduced the magnitude of the lesions. Thus, half of the metastases in this experimental group were detectable only by microscopic analysis (Fig. $4 \mathrm{~B})$. As it was observed for the primary breast tumors, 


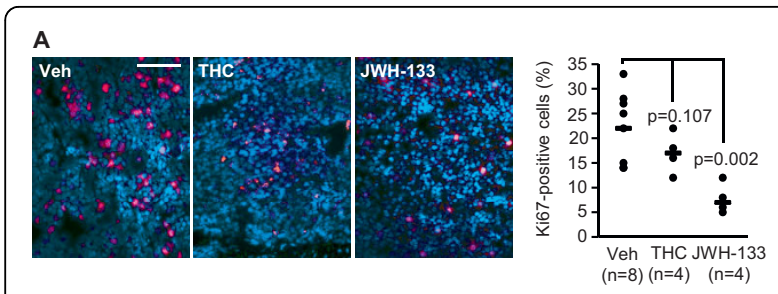

B
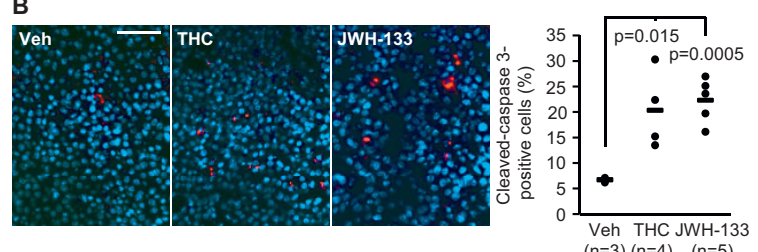

C
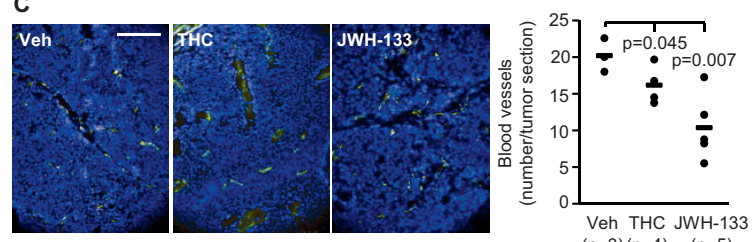

D
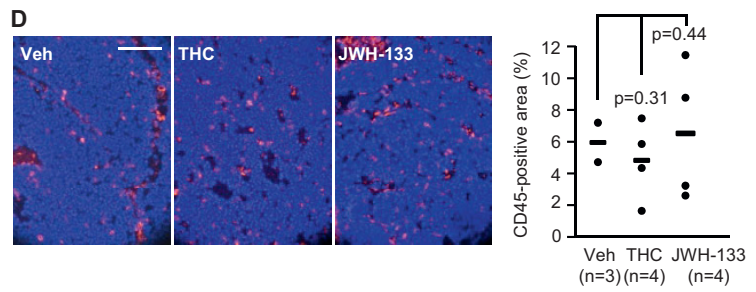

Figure 3 Cannabinoids inhibit cancer cell proliferation, induce cancer cell apoptosis, and impair tumor angiogenesis in vivo. (A) Ki67-positive cells (red), (B) active caspase-3-positive cells (red), (C) CD31-positive cells (green) and (D) CD45-positive area in the tumors. Scale bars: A, $60 \mu \mathrm{m} ; \mathrm{B}, 40 \mu \mathrm{m} ; \mathrm{C}$ and D, $100 \mu \mathrm{m}$. Cell nuclei are in blue. Quantifications of Ki67-positive cells (A), active caspase3-positive cells (B), the number of blood vessels (C) and CD45positive area (D) in the tumors are shown in the corresponding graphs.

cannabinoid treatment did not alter the histopathology of the metastases, and the three experimental groups presented similar solid adenocarcinomas (Additional file 1: Fig. S1C). No sign of metastasis was detected in any of the other organs analyzed (brain, spleen, liver, kidneys -by histological analysis- and bones -by X-rays) in any of the experimental groups (data not shown).

Degradation of the extracellular matrix is a crucial step in the metastatic process, especially during tumor cell intravasation and extravasation [10]. Matrix metalloproteinases (MMPs) have long been associated with this process owing to their ability to degrade the components of the extracellular matrix. To analyze whether cannabinoid administration affects MMP activity we conducted gelatin zymographies. MMP2 activity was decreased in THCand JWH-133-treated tumors, while MMP9 activity was

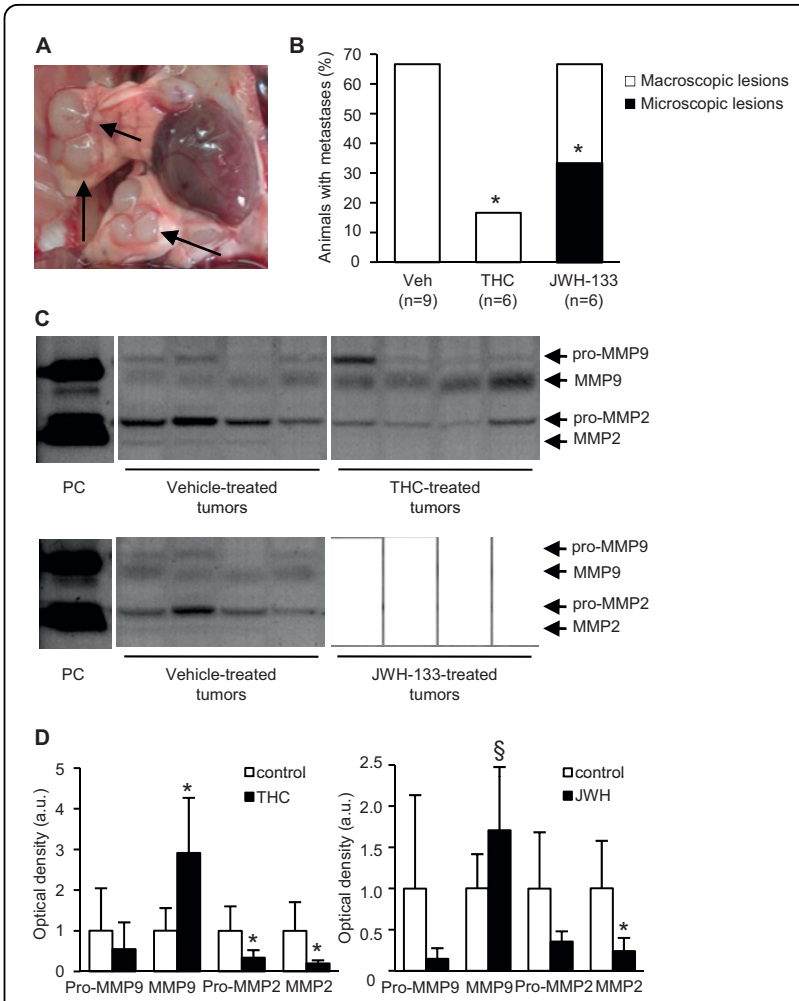

Figure 4 Cannabinoids inhibit breast cancer metastasis to the lungs in vivo. (A) Metastatic lung nodules (pointed by arrows). (B) Percentage of animals with lung metastases. Macroscopic metastases were visible to the naked eye and microscopic lesions were detectable only by H\&E staining. These latter lesions were found only in JWH-133-treated animals. (C) Gelatin zymographies of vehicle- and cannabinoid-treated tumors. Four representative tumors are shown per experimental group. PC: positive control (conditioned medium of $\mathrm{H} 71080$ cells stimulated with the phorbol ester PMA). Arrows point to the latent (pro-MMP) and active MMP bands according to the positive control and the expected MMP molecular weights. Non-contiguous parts of the same gel are shown. Graphs show the densitometric analysis of MMP2 and MMP9 activities. Data are expressed in arbitrary units. ${ }^{*}, p<0.05$ vs vehicle-treated tumors; $\S, p=0.068$ vs vehicle-treated tumors.

enhanced by cannabinoid treatment (Fig. 4C). This THCand JWH-133-induced reduction in MMP2 activity was accompanied by a decrease in MMP2 mRNA levels (Additional file 1: Fig. S4A). Conversely, cannabinoids did not change the amount of MMP9 transcripts (Additional file 1: Fig. S4A) and enhanced its protein levels (Additional file 1: Fig. S4B), indicating that they regulate MMP9 post-transcriptionally.

\section{Akt downregulation is involved in cannabinoid antitumoral action}

We next aimed at characterizing the mechanism underlying cannabinoid antitumoral effect. It is well established that several types of human cancers are associated with deregulation of signaling via ErbB 
members [1]. In particular, ErbB2 overexpression correlates, for instance, with tumor size, increased metastatic potential, and higher histological grade, implying that ErbB2 confers a strong proliferative and survival advantage to tumor cells [11]. To assess whether cannabinoids modulate the expression of endogenous ErbB2 and of the rat ErbB2 ortologue neu, which is ectopically expressed in our animal model, we conducted real-time quantitative PCR determinations upon THC and JWH133 treatment. However, no significant changes were detected (Fig. 5A).

A central intracellular signaling pathway activated by ErbB2 is the PI3K/Akt pathway, whose importance in breast cancer is corroborated by clinical studies showing Akt activation in most ErbB2-overexpressing tumors [11]. We observed a decrease in Akt activation in THCtreated MMTV-neu tumors (Fig. 5B) as well as diminished levels of phosphorylated $\mathrm{S} 6$ ribosomal protein [a read-out for activation of the Akt/mammalian target of rapamycin (mTOR) pathway [12]] (Fig. 5C). To
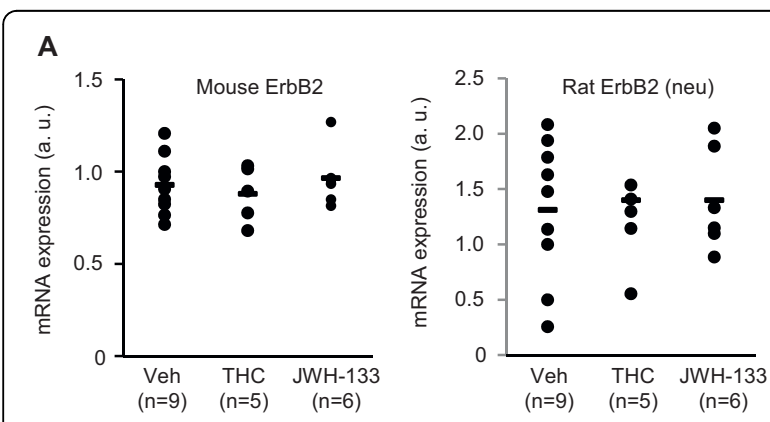

B
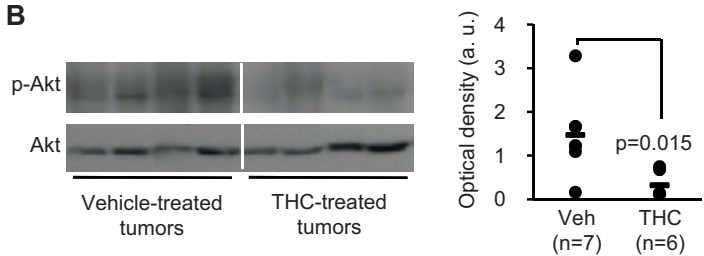

C
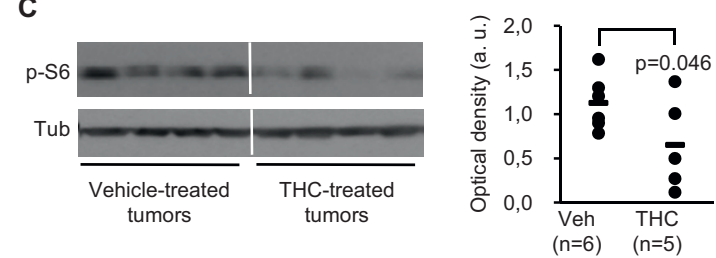

Figure 5 THC inhibits Akt in vivo. (A) Mouse ErbB2 and (B) rat ErbB2 (neu transgene) mRNA expression in vehicle- and cannabinoid-treated tumors as determined by real-time quatitative PCR. (B and C) Western blot and densitometric analysis of phosphoAkt (B) and phospho-S6 ribosomal protein (C) levels in MMTV-neuderived tumors treated with vehicle or THC. Eight representative tumors are shown. Total Akt (B) and $\alpha$-tubulin (C) were used for normalization. Non-contiguous parts of the same gel are shown. Optical densities are expressed in arbitrary units. determine the importance of Akt inhibition in cannabinoid antitumoral action we conducted different experiments with the cell line $\mathrm{N} 202.1 \mathrm{~A}$, that was isolated from a MMTV-neu breast tumor [13]. THC and JWH-133 decreased N202.1A cell proliferation (Fig. 6A) in a $\mathrm{CB}_{2}$ receptor-dependent manner, as indicated by the prevention of cannabinoid action exerted by the $\mathrm{CB}_{2}$ receptor-selective antagonist SR144528 but not by the $\mathrm{CB}_{1}$ receptor-selective antagonist SR141716 (Fig. 6B). Likewise, the growth rate of N202.1A-derived xenografts was significantly diminished by THC and JWH-133, and this effect was prevented by SR144528 (Fig. 6C). THC also decreased cell proliferation of two different ErbB2overexpressing breast cancer cell lines of human origin (Additional file 1: Fig. S5), suggesting that human ErbB2-positive breast tumor cells may be sensitive to cannabinoid antitumoral action as well. N202.1A cells showed a dose-dependent reduction in Akt activation (Fig. 6D). Of interest, overexpression of a myristoylated (i.e. constitutively activated) form of Akt (Fig. 6E) prevented THC antiproliferative effect (Fig. 6F). To further support the importance of Akt in cannabinoid antitumoral action, subcutaneous xenografts were generated in nude mice with N202.1A cells stably expressing myristoylated Akt or the corresponding empty vector (pBABE). As shown in Fig. 6G (left panel), THC significantly reduced the growth of pBABE-transfected N202.1A-derived tumors. In contrast, overexpression of activated Akt prevented THC effect on tumor progression (Fig. 6G, right panel). The same effect was observed with JWH-133 (Fig. 6H).

\section{Discussion}

Aberrant ErbB2 expression and/or function yield highly aggressive tumors, with increased resistance to conventional chemotherapies and poor outcomes. Although the use of the anti-ErbB2 monoclonal antibody Trastuzumab markedly improves the survival of these patients, only $25 \%$ of them respond to this treatment and most of the responders eventually relapse [14]. Moreover, the use of this antibody has been associated with important cardiotoxic side effects (severe congestive heart failure and decrease in left ventricular ejection fraction) [14]. Consequently, extensive efforts should be made to find novel agents for the treatment of ErbB2-positive breast tumors. Our results demonstrate that, in spontaneously aroused ErbB2-overexpressing breast tumors, cannabinoids inhibit tumor generation, growth, vascularization, and metastasis. Although a cannabinoid-based monotherapy might be potentially effective for ErbB2-positive breast tumors, it would be interesting to analyze the effect of these compounds in combination with other anticancer treatments. Thus, it is worth noting that Trastuzumab, the most relevant targeted therapy for 
A

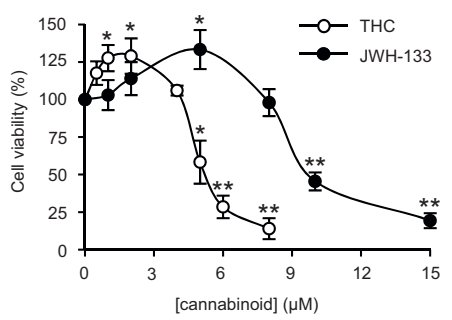

C

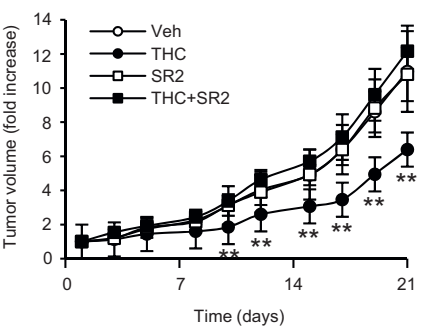

D

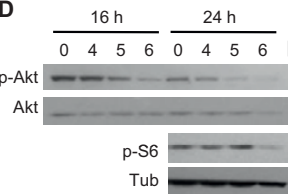

G

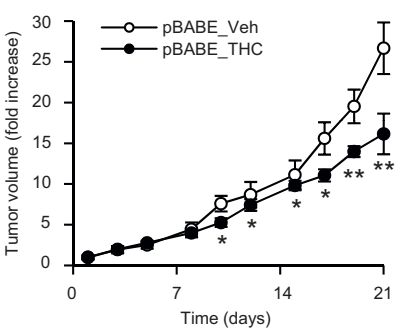

H

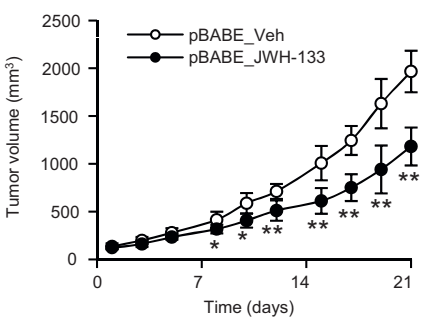

B
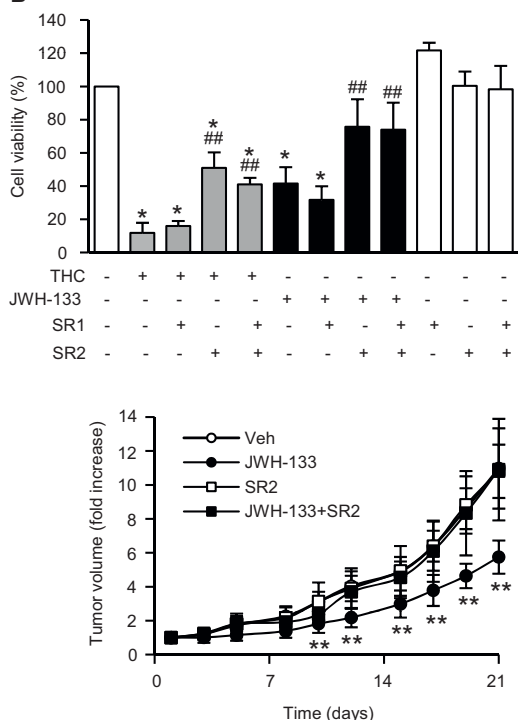

$\mathbf{F}$
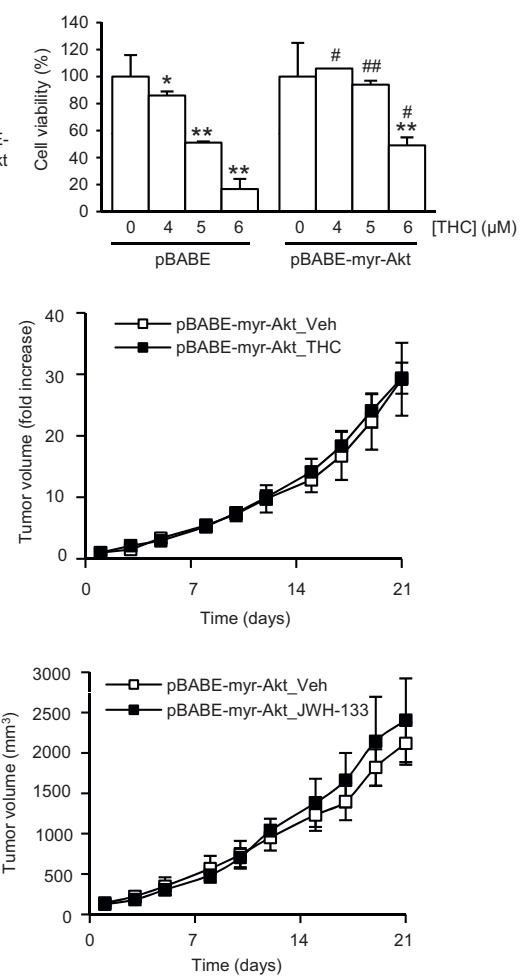

Figure 6 Akt downregulation is involved in cannabinoid antitumoral action. (A and B) Viability of N202.1A cells in response to (A) increasing concentrations of THC or JWH-133 or (B) $6 \mu \mathrm{M}$ THC or $10 \mu \mathrm{M}$ JWH-133 with or without $2 \mu \mathrm{M}$ SR141716 (SR1) and/or SR144528 (SR2) for 48 h. Data are expressed as \% of vehicle-treated cells, set at 100\%. (C) Growth of N202.1 A-derived xenografts treated with THC (left panel) or JWH-133 (right panel) with or without SR2. (D) Phospho-Akt and phospho-S6 ribosomal protein (p-S6) levels in N202.1A cells challenged with THC, as determined by Western blot. Total Akt and $\alpha$-tubulin levels were used for normalization. (E) Phospho-Akt and total Akt levels in N202.1A cells retrovirally transduced with myristoylated Akt (pBABE-Myr-Akt) or the corresponding empty vector (pBABE). (F) Cell viability of pBABE- or myr-Akt-transduced N202.1 A cells in response to THC exposure for $72 \mathrm{~h}$. (G and H) Time course of the volume of pBABE-N202.1 A-derived (left panels) and myr-AktN202.1 A-derived (right panels) tumors treated with THC (G), JWH-133 (H) or the corresponding vehicle. ${ }^{*}, p<0.05 ;{ }^{* *}, p<0.01$ vs vehicle-treated cells or tumors; \#, $p<0.05$; \#\#, $\mathrm{p}<0.01$ vs cannabinoid alone-treated cells (B) or vs THC-treated pBABE-transduced cells (F). 
ErbB2-positive tumors so far, has a modest median overall response when used as a first-line agent, an efficacy that is clearly enhanced when used in combination with other chemotherapeutic agents [14]. Additionally, Akt overactivation has been detected in a significant percentage of primary human breast cancers, in which it is associated to enhanced resistance to Trastuzumab $[14,15]$. Our results show that downregulation of Akt is involved in cannabinoid antitumoral action. This kinase is the central node of the PI3K/Akt/mTOR signaling pathway, that activates crucial processes such as cell survival, cell growth, cell proliferation, angiogenesis, and cell migration and invasion [12]. This pathway is therefore an attractive target for anticancer agents and, as a matter of fact, clinical trials have been/are being conducted with mTOR, PI3K and Akt inhibitors [3].

The antitumoral potential of cannabinoids has been documented both in vitro and in animal models of cancer $[7,8]$. These compounds inhibit breast cancer cell proliferation in vitro through processes that include cell cycle arrest [16-21], hormone and growth-factor receptor modulation $[18,22,23]$, and apoptosis induction $[17,20,21]$. The in vivo approaches followed so far have been mostly based on xenograft models [20,21], which are helpful but limited tools. These models rely on the propagation of cancer cell lines in immunodeficient mice at ectopic or orthotopic sites and lack crucial features of patients' tumors such as the actual tumor architecture and the interactions with the tumor microenvironment (including non-cancerous surrounding tissue, vasculature and immune cells) and diminished genetic heterogeneity [24]. In contrast to xenografted animals, in the mutant mice used in this study tumors appear spontaneously and after long latency periods, recruit and generate blood vessels, and penetrate the vasculature giving rise to distant metastases [9]. These features parallel the human pathology much more closely and make the MMTV-neu mice a clinically relevant model of ErbB2-driven breast cancer.

Remarkably, this is, to the best of our knowledge, the first report supporting that cannabinoids hamper not only tumor growth but also tumor generation. Recently, Qamri and coworkers and DuBois and coworkers, by using two different genetic models of cancer, demonstrated that JWH-133 delays the appearance of breast tumors [21] and that the loss of $\mathrm{CB}_{1}$ receptors accelerates intestinal adenoma growth [25], respectively, and Izzo et al. observed that high endocannabinoid levels and the cannabinoid agonist HU-210 reduce the development of precancerous lesions in the mouse colon [26]. These and our data suggest that the endocannabinoid system has a physiological protective role against tumorigenesis, in line with the general idea that this system contributes to maintain homeostasis in health and disease [6].
Data presented herein show that cannabinoids modulate MMP activity. In particular, we report an inhibition of MMP2 and an activation of MMP9 by cannabinoids. Although MMPs have been traditionally associated to metastasis due to their ability to degrade the extracellular matrix, it has been recently shown that several members of this family provide a protective effect in different stages of cancer progression [27]. One of these antitumoral MMPs is MMP9, which is activated by cannabinoids in our system. Although this MMP may promote the angiogenic switch in some experimental tumors [e.g. [28]], clinical studies have established a correlation between MMP9 overexpression and good prognosis in breast cancer [29]. This protective effect might derive from its capacity to generate angiogenesis inhibitors such as angiostatin and tumstatin [27]. The inhibition of MMP2 by cannabinoids shown here, in line with that previously reported by Bifulco and coworkers in thyroid cancer cells [30] and our group in gliomas [31], may be of special relevance considering that high tumor levels of this metalloproteinase have been correlated with poor prognosis in breast cancer [32]. In addition, enhanced levels of MMP2 in breast tumors are associated with ErbB2 gene amplification and/or overexpression [33]. Moreover, Massagué and coworkers have recently identified MMP2 as one of the genes of the signature that mediates breast cancer metastasis to the lungs [34], the targeted metastatic organ in our animal model.

Potential antitumoral therapies based on the use of cannabinoids might be limited by their well known psychotropic actions such as dizziness, dry mouth, tiredness, muscle weakness, euphoria, myalgia and palpitations [6,35]. Although the benefit/risk ratio is potentially high for cannabinoid-based therapies, different strategies should be taken to avoid or at least minimize their side effects. Since most -if not all- of the psychoactive effects of cannabinoids are produced by the activation of central $\mathrm{CB}_{1}$ receptors [5,6], one reasonable approach would be targeting $\mathrm{CB}_{2}$ receptors selectively. Here, we have demonstrated that the $\mathrm{CB}_{2}$ selective agonist JWH-133 is as effective as THC (a $\mathrm{CB}_{1} / \mathrm{CB}_{2}$-mixed agonist) in reducing tumor generation and progression. Moreover, our results also (i) show that an elevated percentage of high grade ErbB2-positive human breast tumors express $\mathrm{CB}_{2}$ receptors, and (ii) that a very low fraction of them express $C_{1}$ receptors. Taken together, these data suggest that activation of $\mathrm{CB}_{2}$ in this particular population of patients would be an efficient strategy to treat breast tumors without triggering psychoactive effects. A correlation between tumor aggressiveness and $\mathrm{CB}_{2}$ receptor expression in breast cancer has been previously reported: tumors lacking estrogen or progesterone receptors, which are associated to low response rates to adjuvant therapies, 
express higher $\mathrm{CB}_{2}$ levels than steroid receptor-positive lesions [17]. Moreover, ErbB2-positive tumors also have increased $\mathrm{CB}_{2}$ receptor mRNA levels compared to their less aggressive and more responsive ErbB2-negative counterparts [17]. Of interest, this receptor is scarcely expressed in non-transformed mammary tissue [data presented here and [17,21]].

\section{Conclusions}

In summary, our results, which were obtained in a clinically relevant animal model of ErbB2-positive breast cancer, suggest that these highly aggressive and low responsive tumors could be efficiently treated with nonpsychoactive $\mathrm{CB}_{2}$-selective agonists without affecting the surrounding healthy tissue.

\section{Methods}

\section{Tissue microarray analysis}

Eighty seven grade 3 invasive breast ductal carcinomas and 6 non-tumoral mammary tissues were fixed in 10\% paraformaldehide (PFA) and embedded in paraffin. Two representative tissue cores (1 $\mathrm{mm}$ of diameter) of each one were included in a tissue microarray. The main clinical pathology and molecular features of this series had been previously reported [36]. Tissue sections were subjected to a heat-induced antigen retrieval step prior to exposure to the primary antibody. Anti$\mathrm{CB}_{1}$ receptor antibody was generously donated by Dr. Ken Mackie, Indiana University, Indiana, and anti- $\mathrm{CB}_{2}$ receptor antibody was from Affinity Bioreagents/ Thermo Fisher Scientific, Rockford, Illinois. ErbB2 expression was evaluated using a HercepTest (Dako, Carpenteria, CA) according to manufacturer's instructions. Immunodetection was performed using the LSAB method (DAKO) with DAB as the chromogen. In negative controls, the primary antibody was omitted or replaced with an irrelevant antibody. Cases were reviewed by two independent pathologists (J.P. and G.M-B) and were scored as positive for cannabinoid receptors when more than $25 \%$ of the neoplastic cell showed intense immunostaining for the corresponding antibody. ErbB2-staining was scored according to HercepTest manufacturer's guidelines: scores 0 and $1+$ were considered as negative, and $2+$ and $3+$ as positive for ErbB2 overexpression.

\section{Animals and treatments}

All procedures involving animals were performed with the approval of the Complutense University Animal Experimentation Committee according to the European official regulations. FVB/N-Tg(MMTVneu)202 Mul/J mice (more commonly designated as MMTV-neu mice) were obtained from The Jackson Laboratory (Bar Harbor, Maine). Females were palpated twice weekly for mammary gland nodules and cannabinoid treatment was started when the first tumor in each animal was detected. $\Delta^{9}$-Tetrahydrocannabinol (THC, The Health Concept, Richelbach, Germany) and JWH-133 (kindly donated by John W. Huffman, Clemson University, South Carolina) were prepared in DMSO $(0.2 \mathrm{mg} / \mu \mathrm{L}$ and $0.02 \mathrm{mg} / \mu \mathrm{L}$, respectively) and diluted in PBS supplemented with $5 \%$ BSA $(100 \mu \mathrm{L} /$ dose for tumors $\leq$ $1000 \mathrm{~mm}^{3}$ and $200 \mu \mathrm{l} /$ dose for tumors $>1000 \mathrm{~mm}^{3}$ ). Cannabinoid peritumoral treatment $(0.5 \mathrm{mg}$ THC or $0.05 \mathrm{mg} \mathrm{JWH-133/animal/day,} \mathrm{twice} \mathrm{a} \mathrm{week)} \mathrm{was} \mathrm{main-}$ tained for 90 days, and only the first tumor in each animal was treated. Tumors were routinely measured during this period with external caliper, and volume was calculated as $(4 \pi / 3) \times(\text { width } / 2)^{2} \times($ length $/ 2)$. At the end of the treatment, animals were sacrificed and tumors and organs were collected. Tumors were divided in four portions for 1) preparation of tissue sections for immunofluorescent staining [frozen in Tissue-Tek (Sakura Finetek Europe, Zoeterwoude, The Netherlands)], 2) preparation of tissue sections for hematoxylin-eosin staining (fixed in buffered 4\% PFA), 3) protein extraction (snap frozen) and 4) RNA isolation (snap frozen), and were stored at $-80^{\circ} \mathrm{C}$ until analysis (except PFA-fixed tumor fractions, that were kept at room temperature). Brain, spleen, liver, kidneys and lungs were fixed in PFA. For xenograft experiments, subcutaneous tumors were induced in 6 week-old athymic female mice (Harlan Interfauna Iberica, Barcelona, Spain) by subcutaneous injection of $5 \times 10^{5} \mathrm{~N} 202.1 \mathrm{~A}$ cells. When tumors reached $c a .100 \mathrm{~mm}^{3}$, they were treated with THC (0.5 mg/animal/day), JWH-133 (50 $\mu \mathrm{g} /$ animal/day), SR144528 (50 $\mu \mathrm{g} / \mathrm{animal} /$ day), a combination of cannabinoid and SR144528 or vehicle for 3 weeks, 3 times a week, measured, and processed as described above. For Akt-related experiments, half of the animals were injected with N202.1A cells stably expressing myristoylated Akt (N202.1A-pBABE-myr-Akt), and the other half with N202.1A cells stably expressing the corresponding empty vector (N202.1A-pBABE). Tumors were treated with THC, JWH-133 or vehicle and processed as described for N202.1A xenografts.

\section{Metastasis detection}

Collected organs were visually analyzed for macroscopic metastases. Microscopic metastases were determined by histological analysis of PFA-fixed paraffin-embedded hematoxylin-eosin stained sections. Radiographs were taken to evaluate the presence of bone metastases using a conventional X-ray equipment (Diagnost 93, Philips Medical Systems, Eindhoven, The Netherlands), and mammography cassette (Kodak MIN-R 2000 screen cassette) and film (Kodak MIN-R S film) (Eastman Kodak Company, Rochester, New York). 


\section{Cell culture and viability}

N202.1A cells were kindly given by Dr. Vincenzo Bronte (Istituto Oncologico Veneto, Padova, Italy). This cell line was established from a MMTV-neu-derived tumor [13]. BT474, MDA-MB-231, MCF-7 and SkBr3 human breast cancer cells, Jurkat human leukemic cells, and U373 human glioblastoma cells were from ATCC-LGC (Barcelona, Spain). All cell lines were maintained in DMEM supplemented with $10 \%$ fetal bovine serum (FBS). Cells were transferred to a low (0.5\%)-FBS medium immediately before cannabinoid challenge. Cell viability was determined by the 3-4,5-dimethylthiazol-2,5diphenyltetrazolium bromide thiazol blue test (Sigma, St. Louis, Missouri) according to manufacturer's instructions.

\section{Plasmids, transfections and infections}

Stable expression of myr-Akt was achieved by retroviral infection. N202.1A cells were transduced for $4 \mathrm{~h}$ with supernatants obtained from Phoenix ecotropic cells previously transfected with a retroviral vector carrying HAtagged myr-Akt (kindly provided by Dr. Pier P. Pandolfi, Harvard University, Boston, Massachusetts) or the corresponding empty construction (pBABE). Infected cells were selected with puromycin.

\section{Immunofluorescence analysis}

Tissue-tek embedded tumor sections were fixed in PFA and incubated with anti- $\mathrm{CB}_{1}$ receptor, anti- $\mathrm{CB}_{2}$ receptor, anti-CD31 (Pharmingen/BD Biosciences, San Jose, California), anti-CD45 (Pharmingen/BD Biosciences), antiKi67 (Neomarkers/Lab Vision, Fremont, California) or anti-cleaved-caspase 3 (Cell Signaling Technology, Danvers, Massachusetts) antibodies. Secondary anti-rabbit antibodies AlexaFluor 594 and AlexaFluor 488 were from Invitrogen (Carlsbad, California). Cell nuclei were stained with Hoescht 33342 (Invitrogen). Fluorescence images were acquired using Metamorph Premier Offline software (Molecular Devices, Sunnyvale, California). Blood vessel size was calculated with ImageJ software.

\section{Real-time quantitative PCR (RTQ-PCR) and reverse- transcriptase PCR (RT-PCR)}

RNA was isolated with Trizol Reagent (Invitrogen), including a DNase digestion step, with the Real Star Kit (Durviz, Valencia, Spain), and cDNA was obtained with Transcriptor Reverse Transcriptase (Roche Applied Science, Penzberg, Germany). The primers used for RTQ-PCR amplification were: mouse $C_{1}$ receptor, sense 5'-GGGCAAATTTCCTTGTAGCA-3', antisense 5'-GGCTCAACGTGACTGAGAAA-3'; mouse $\mathrm{CB}_{2}$ receptor, sense 5'-ATTCAGGAGATCTGTTAAGA CAAGG-3', antisense 5'-GACATCTATGAAGTTGAG GCAGTG-3'; mouse MMP2, sense 5'- GCGCTTTTC-
TCGAATCCAT-3', antisense 5'-GGGTATCCATCTC CATGCTC-3'; mouse MMP9, sense 5'-ACGACATA GACGGCATCCA-3', antisense 5' - GCTGTGGTTCA -GTTGTGGTG-3'; rat ErbB2 (neu), sense 5'-GCTCA GAGACCTGCTTTGGA-3', antisense 5'-AGGAGGAC GAGTCCTTGTAGTG-3'; mouse ErbB2, sense 5'AACAGCTCGGAGACCTGCTA-3', antisense 5'GTAGTGGGCACAAGCCTCA-3'. Probes were from the Universal Probe Library (Roche Applied Science). Multispecies 18S RNA was used as reference (sense $5^{\prime}$ GCTCTAGAATTACCACAGTTATCCAA-3', antisense 5' - AAATCAGTTATGGTTCCTTTGGTC-3'). The primers use for RT-PCR were: mouse MMP2, sense $5^{\prime}$ TCTGCGATGAGCTTAGGGAAAC-3', antisense 5' GACATACATCTTTGCAGGAGACAAG-3'; mouse MMP9, sense 5'-GGACGACGTGGGCTACGT-3', antisense 5' - CACGGTTGAAGCAAAGAAGGA-3'. GAPDH was used as reference (sense 5'-GGGAAGCT CACTGGCATGGCCTTCC-3', antisense 5'-CATGTG GGCCATGAGGTCCACCAC-3').

\section{Western blot analysis}

Cell lysates from tumors and cell lines were subjected to SDS-PAGE, and proteins transferred onto polyvinylidene fluoride membranes. Blots were incubated with the following antibodiess: anti- $\mathrm{CB}_{1}$ receptor, anti- $\mathrm{CB}_{2}$ receptor (Affinity Bioreagents), anti-MMP9 (Chemicon International INC, Temecula, California), anti-ErbB2 (Santa Cruz Biotechnology, Santa Cruz, California), anti-phospho-Akt (Ser473), anti-Akt, anti-phospho-S6 ribosomal protein (Cell Signaling) and anti- $\alpha$-tubulin (Sigma). Luminograms were obtained with the Amersham Enhanced Chemiluminescence Detection Kit (GE Healthcare, Uppsala, Sweden) and densitometric analysis was performed with Quantity One software (Bio-Rad).

\section{MMP activity assay}

MMP2 (Gelatinase A) and MMP9 (Gelatinase B) activities were determined by gelatin zymography. Briefly, SDS-PAGE were run in the presence of $0.1 \%$ gelatin, washed with a $2.5 \%$ Triton $\mathrm{X}-100$ containing buffer, and incubated overnight at $37^{\circ} \mathrm{C}$ in $50 \mathrm{mM}$ Tris $\mathrm{pH} 7.5,150$ $\mathrm{mM} \mathrm{NaCl}, 10 \mathrm{mM} \mathrm{CaCl}$, $0.1 \%$ Triton X100. Gels were then stained with Coomasie Blue and digested bands quantified by densitometric analysis as described above.

\section{Statistical analysis}

ANOVA with a post hoc analysis by the Student-Newman-Keuls' test was routinely used. For the analysis of metastases and the number of tumors per animal, a Pearson $\mathrm{X}^{2}$ test was used. To determine the correlation between immunohistochemical $\left(\mathrm{CB}_{1}\right.$ and $\mathrm{CB}_{2}$ expression) and clinical pathology (ErbB2) data, the $\mathrm{X}^{2}$ test with Yates correction, or Fisher's exact test, was used. 
The SPSS for Windows program (SPSS, Inc., Chicago, IL, version 17.0) was used for this analysis. All P-values were two-sided. Unless otherwise stated, data are expressed as mean \pm s.e.m.

\section{Additional material}

Additional file 1: Figures S1-S5. Supplemental Figure 1: MMTV-neu mice spontaneously develop breast tumors and lung metastases. Supplemental Figure 2: Cannabinoids inhibit breast tumor growth in vivo Supplemental Figure 3: MMTV-neu-derived tumors express cannabinoid receptors Supplemental Figure 4: Cannabinoids modulate the expression of MMP2 and MMP9 Supplemental Figure 5: Human ErbB2-positive breast cancer cell lines are sensitive to cannabinoids

\section{Acknowledgements}

We are indebted to Dr. Vincenzo Bronte (Istituto Oncologico Veneto, Fondazione IRCCS, Padova, Italy) for the kind donation of the N202.1A cells. We would also like to thank the members of our laboratories, especially María Salazar, Sonia Hernández-Tiedra and Marta Cifali, for technical support and critical discussion on this work. This study was supported by grants from Fondo de Investigaciones Sanitarias (PI080253 to CS and PI086080971 to JP), Ministerio de Ciencia e Innovación (SAF2006-00918 to MG, SAF200763075 to GMB and SAF2008-00649 to SM), RETICS (RD06/0020/0013 to JP), Fundación Mutua Madrileña (to CS and to GMB), Consejería de Innovación Junta de Andalucía (to JP), the Marató TV3 Foundation (053132 to SM) and GW Pharmaceuticals/Otsuka Pharmaceuticals (to CS). MMC was the recipient of a Fellowship from Fundación Ferrer para la Investigación.

\section{Author details}

${ }^{1}$ Dept. Biochemistry and Molecular Biology I, School of Biology, Complutense University, Madrid, Spain. ${ }^{2}$ Dept. Immunology and Oncology, Centro Nacional de Biotecnología, Madrid, Spain. ${ }^{3}$ Dept. Biochemistry, School of Medicine, Universidad Autónoma-Instituto de Investigaciones Biomédicas "Alberto Sols", IdiPaz, Madrid, Spain. ${ }^{4}$ Dept. Animal Surgery and Medicine, School of Veterinary, Complutense University, Madrid, Spain. ${ }^{5}$ Servicio de Anatomía Patológica, Hospital Virgen del Rocío, Seville, Spain. ${ }^{6}$ Current Address: MMC, Dept. Pathology, University of Cambridge, United Kingdom; CC, Dept. Life Sciences, The Open University, Milton Keynes, UK.

\section{Authors' contributions}

MMC participated in the design of the experiments and carried out animal treatments, sample collection and processing (MMP activity assays, quantitative PCRs, Western blot analyses, immunofluorescence experiments and cell culture approaches) and statistical analyses. CA and EP-G participated in animal treatments, sample collection, quantitative PCRs and Western blot analyses. EM participated in the design of the study and carried out immunofluorescence experiments. CC participated in animal treatments and immunofluorescence experiments. JMF carried out the histopathological analyses of animal tumors and metastases. GM-B performed and analyzed the tissue microarrays of human samples. IG-R analyzed metastases in MMTV-neu mice by magnetic resonance imaging. JP carried out the histopathological characterization of human breast samples. SM participated in the design of the study. MG contributed to the conception and design of the study and critically revised the manuscript. CS conceived the study, elaborated its design, coordinated the research and wrote the manuscript. All authors read and approved the final manuscript.

\section{Competing interests}

The authors declare that they have no competing interests.

Received: 26 January 2010 Accepted: 22 July 2010

Published: 22 July 2010
References

1. Baselga J, Swain SM: Novel anticancer targets: revisiting ERBB2 and discovering ERBB3. Nat Rev Cancer 2009, 9:463-475.

2. Ursini-Siegel J, Schade B, Cardiff RD, Muller WJ: Insights from transgenic mouse models of ERBB2-induced breast cancer. Nat Rev Cancer 2007, 7:389-397

3. Di Cosimo S, Baselga J: Targeted therapies in breast cancer: where are we now? Eur J Cancer 2008, 44:2781-2790.

4. Hynes NE, Lane HA: ERBB receptors and cancer: the complexity of targeted inhibitors. Nat Rev Cancer 2005, 5:341-354.

5. Di Marzo V: Targeting the endocannabinoid system: to enhance or reduce? Nat Rev Drug Discov 2008, 7:438-455.

6. Pertwee RG: Emerging strategies for exploiting cannabinoid receptor agonists as medicines. Br J Pharmacol 2009, 156:397-411.

7. Guzman M: Cannabinoids: potential anticancer agents. Nat Rev Cancer 2003, 3:745-755.

8. Sarfaraz S, Adhami VM, Syed DN, Afaq F, Mukhtar H: Cannabinoids for cancer treatment: progress and promise. Cancer Res 2008, 68:339-342.

9. Guy CT, Webster MA, Schaller M, Parsons TJ, Cardiff RD, Muller WJ: Expression of the neu protooncogene in the mammary epithelium of transgenic mice induces metastatic disease. Proc Natl Acad Sci USA 1992 89:10578-10582.

10. Chambers AF, Groom AC, MacDonald IC: Dissemination and growth of cancer cells in metastatic sites. Nat Rev Cancer 2002, 2:563-572.

11. Moasser MM: The oncogene HER2: its signaling and transforming functions and its role in human cancer pathogenesis. Oncogene 2007, 26:6469-6487.

12. Manning BD, Cantley LC: AKT/PKB signaling: navigating downstream. Cell 2007, 129:1261-1274

13. Nanni P, Pupa SM, Nicoletti G, De Giovanni C, Landuzzi L, Rossi I, Astolfi A, Ricci C, De Vecchi R, Invernizzi AM, et al: p185(neu) protein is required for tumor and anchorage-independent growth, not for cell proliferation of transgenic mammary carcinoma. Int J Cancer 2000, 87:186-194.

14. Dean-Colomb W, Esteva FJ: Her2-positive breast cancer: herceptin and beyond. Eur J Cancer 2008, 44:2806-2812.

15. Hutchinson JN, Jin J, Cardiff RD, Woodgett JR, Muller WJ: Activation of Akt1 (PKB-alpha) can accelerate ErbB-2-mediated mammary tumorigenesis but suppresses tumor invasion. Cancer Res 2004, 64:3171-3178.

16. Caffarel MM, Moreno-Bueno G, Cerutti C, Palacios J, Guzman M, MechtaGrigoriou F, Sanchez C: JunD is involved in the antiproliferative effect of Delta(9)-tetrahydrocannabinol on human breast cancer cells. Oncogene 2008, 27:5033-5044.

17. Caffarel MM, Sarrio D, Palacios J, Guzman M, Sanchez C: Delta9tetrahydrocannabinol inhibits cell cycle progression in human breast cancer cells through Cdc2 regulation. Cancer Res 2006, 66:6615-6621.

18. De Petrocellis L, Melck D, Palmisano A, Bisogno T, Laezza C, Bifulco M, Di Marzo V: The endogenous cannabinoid anandamide inhibits human breast cancer cell proliferation. Proc Natl Acad Sci USA 1998, 95:8375-8380.

19. Laezza C, Pisanti S, Crescenzi E, Bifulco M: Anandamide inhibits Cdk2 and activates Chk1 leading to cell cycle arrest in human breast cancer cells. FEBS Lett 2006, 580:6076-6082.

20. Ligresti A, Moriello AS, Starowicz K, Matias I, Pisanti S, De Petrocellis L Laezza C, Portella G, Bifulco M, Di Marzo V: Antitumor activity of plant cannabinoids with emphasis on the effect of cannabidiol on human breast carcinoma. J Pharmacol Exp Ther 2006, 318:1375-1387.

21. Qamri Z, Preet A, Nasser MW, Bass CE, Leone G, Barsky SH, Ganju RK: Synthetic cannabinoid receptor agonists inhibit tumor growth and metastasis of breast cancer. Mol Cancer Ther 2009, 8:3117-3129.

22. Melck D, De Petrocellis L, Orlando P, Bisogno T, Laezza C, Bifulco M, Di Marzo V: Suppression of nerve growth factor Trk receptors and prolactin receptors by endocannabinoids leads to inhibition of human breast and prostate cancer cell proliferation. Endocrinology 2000, 141:118-126.

23. Melck D, Rueda D, Galve-Roperh I, De Petrocellis L, Guzman M, Di Marzo V: Involvement of the CAMP/protein kinase A pathway and of mitogenactivated protein kinase in the anti-proliferative effects of anandamide in human breast cancer cells. FEBS Lett 1999, 463:235-240.

24. Frese KK, Tuveson DA: Maximizing mouse cancer models. Nat Rev Cancer 2007, 7:645-658 
25. Wang D, Wang H, Ning W, Backlund MG, Dey SK, DuBois RN: Loss of cannabinoid receptor 1 accelerates intestinal tumor growth. Cancer Res 2008, 68:6468-6476.

26. Izzo AA, Aviello G, Petrosino S, Orlando P, Marsicano G, Lutz B, Borrelli F, Capasso R, Nigam S, Capasso F, Di Marzo V: Increased endocannabinoid levels reduce the development of precancerous lesions in the mouse colon. J Mol Med 2008, 86:89-98.

27. Lopez-Otin C, Matrisian LM: Emerging roles of proteases in tumour suppression. Nat Rev Cancer 2007, 7:800-808.

28. Mira E, Lacalle RA, Buesa JM, de Buitrago GG, Jimenez-Baranda S, GomezMouton C, Martinez AC, Manes S: Secreted MMP9 promotes angiogenesis more efficiently than constitutive active MMP9 bound to the tumor cell surface. J Cell Sci 2004, 117:1847-1857.

29. Scorilas A, Karameris A, Arnogiannaki N, Ardavanis A, Bassilopoulos P, Trangas T, Talieri M: Overexpression of matrix-metalloproteinase- 9 in human breast cancer: a potential favourable indicator in node-negative patients. Br J Cancer 2001, 84:1488-1496.

30. Pisanti S, Borselli C, Oliviero O, Laezza C, Gazzerro P, Bifulco M: Antiangiogenic activity of the endocannabinoid anandamide: correlation to its tumor-suppressor efficacy. I Cell Physiol 2007, 211:495-503.

31. Blazquez C, Salazar M, Carracedo A, Lorente M, Egia A, Gonzalez-Feria L, Haro A, Velasco G, Guzman M: Cannabinoids inhibit glioma cell invasion by down-regulating matrix metalloproteinase-2 expression. Cancer Res 2008, 68:1945-1952.

32. Decock J, Paridaens R, Cufer T: Proteases and metastasis: clinical relevance nowadays? Curr Opin Oncol 2005, 17:545-550.

33. Jezierska A, Motyl T: Matrix metalloproteinase-2 involvement in breast cancer progression: a mini-review. Med Sci Monit 2009, 15:RA32-40.

34. Minn AJ, Gupta GP, Siegel PM, Bos PD, Shu W, Giri DD, Viale A, Olshen AB, Gerald WL, Massague J: Genes that mediate breast cancer metastasis to lung. Nature 2005, 436:518-524.

35. Guzman M, Duarte MJ, Blazquez C, Ravina J, Rosa MC, Galve-Roperh I, Sanchez C, Velasco G, Gonzalez-Feria L: A pilot clinical study of Delta9tetrahydrocannabinol in patients with recurrent glioblastoma multiforme. Br J Cancer 2006, 95:197-203.

36. Natrajan R, Lambros MB, Rodriguez-Pinilla SM, Moreno-Bueno G, Tan DS, Marchio C, Vatcheva R, Rayter S, Mahler-Araujo B, Fulford LG, et al: Tiling path genomic profiling of grade 3 invasive ductal breast cancers. Clin Cancer Res 2009, 15:2711-2722.

doi:10.1186/1476-4598-9-196

Cite this article as: Caffarel et al:: Cannabinoids reduce ErbB2-driven breast cancer progression through Akt inhibition. Molecular Cancer 2010 9:196.

\section{Submit your next manuscript to BioMed Central and take full advantage of:}

- Convenient online submission

- Thorough peer review

- No space constraints or color figure charges

- Immediate publication on acceptance

- Inclusion in PubMed, CAS, Scopus and Google Scholar

- Research which is freely available for redistribution

Submit your manuscript at www.biomedcentral.com/submit
Ciomed Central 